\title{
Study on Reducing the Stress of Wearing a Mask through Deep Breathing
}

\author{
Zhixing Tian', Bong-Young Kim² and Myung-Jin Bae ${ }^{3 *}$ \\ ${ }^{1}$ Soong-sil University, Department of Information and telecommunication Engineering, Seoul, 06978, Korea. \\ ORCID ID: 0000-0003-3882-2459 \\ ${ }_{2}^{2}$ Soong-sil University, Department of Information and telecommunication Engineering, Seoul, 06978, Korea. \\ ORCID ID: 0000-0002-3553-039X \\ ${ }^{3}$ Soong-sil University, Department of Information and telecommunication Engineering, Seoul, 06978, Korea. \\ ORCID ID: 0000-0002-7585-0400
}

\begin{abstract}
:
With the large-scale outbreak of the COVID epidemic worldwide, the number of people using protective masks has increased rapidly, However, problems such as stress and hypoxia symptoms caused by wearing masks for long periods of time have not received enough attention. In fact, these may greatly harm human health and weaken the protective effect of the mask. So, research on stress reduction is also important. Deep breathing is a classic and practical method to reduce stress and overcome nervousness. It has been widely recognized in physiology and psychology. This paper explains the research on the application of deep breathing method to reduce the stress induced by wearing a mask for a long time, explains the causes of stress, and principle of deep breathing method to relieve stress. Finally, the experiment was obtained through the SDNN analysis experiment of HRV. in conclusion. This research not only provides a good way for many people who have to wear masks for a long time to relieve stress and reduce the symptoms of hypoxia, but also suggestions for the public to use mask more scientifically and safely.
\end{abstract}

Keyword: Mask, stress, deep breathing, hypoxia, HRV, COVID 19, SDNN

\section{INTRODUCTION}

The worldwide outbreak of COVID 19 has convert many countries into severe epidemic areas. Home quarantine, hand sanitizer, and masks have become public means of epidemic prevention. However, wearing protective masks for a long time causes stress to people, and this stress response will damage human's physical and mental health. Especially, it may reduce their immunity, which will increase the risk of infection. However, the most direct way to reduce the stress induced by wearing a mask is to take off the mask and breathe normally and take some time off. As the outbreak has worsened, most people have opted for home quarantine, but some still need to work in offices and travel by car. For example, the medical workers who are in the forefront of the fighting, the government administrators, the international students who are flying home by plane and so on. They usually work and move in confined spaces, and they are more likely to contact patients. If they remove their masks, they will increase their probability of being infected. Therefore, it is not suitable to take off the mask to breathe normally in many activities [1][2].

The study found that the deep breathing method can alleviate the stress induced by wearing a mask and simultaneously solve the problem of hypoxia when wearing a mask for a long time. This classic and simple method has been used to reduce stress and overcome tension in the past. It has been extensively certified in physiology and psychology. It is still very effective in reducing the stress increase and hypoxia caused by masks [3] [4] [5].

In Chapter 2, we explained the causes of the induced stress caused by wearing a mask for a long time. In Chapter 3, proposed the deep breathing method and explained the principle that it can relieve the stress. In Chapter 4, introduce a method for measuring the stress, that is, using HRV analysis to correlate with the stress. In Chapter 5, describe an experiment in which the stress is changed by measuring heart rate during deep breathing, and the experimental results are obtained. In Chapter 6, conclusion.

\section{CAUSES AND HARM OF STRESS INDUCED BY WEARING A MASK}

Wearing a mask causes stress for two reasons. On the one hand, the protective mask has strong air tightness and large breathing resistance, and the brain will feel that breathing is more strenuous than usual. So, this negative factor will cause psychological stress. On the other hand, due to the limited permeability of the mask, the emission of carbon dioxide and the inhalation of oxygen are hindered, and the concentration of oxygen in the inhaled air decreases, resulting in hypoxia. Hypoxia is a source of stimuli that causes more stressful responses. This is also the root cause of the increase in people's stress [6] [7]. 
In summary, the breathing is blocked due to wearing a mask. Generally, when the gas passes through the respiratory tract, it is subject to resistance. In respiratory physiology, the resistance of the respiratory tract to airflow during inhalation and exhalation is called as airway resistance [8]. Airway resistance is defined as the pressure difference produced by the unit flow in the airway, that is:

$$
\begin{aligned}
& R_{A W}=\frac{\Delta P}{\dot{V}} \\
& \text { Where } \Delta P=P_{A T M}-P_{A}, \text { and } \dot{V}=v * S, \\
& \text { Thus, } R_{A W}=\frac{P_{A T M}-P_{A}}{v * S},
\end{aligned}
$$

$R_{A W}$ is Airway Resistance, $P_{A T M}$ is Atmospheric Pressure, $P_{A}$ is Alveolar Pressure. $v$ is flow velocity, and $s$ is crosssectional vector area.

First, according to the equation, the airway resistance is directly proportional to pressure difference and inversely proportional to flow velocity and cross-sectional area. The airway can be assumed to be a cylindrical tube that two ends connected to the outside and the lungs. When wearing a mask, it is equivalent to reducing the cross-sectional area $(s)$ of the externally connected segments and reducing the flow velocity $(v)$. And because of the blocking of the mask, the pressure $\left(P_{A T M}\right)$ at the end connected to the outside world increases, and eventually the airway resistance $\left(R_{A W}\right)$ increases, which makes our breathing hard and increases our psychological stress.

During inhalation, the air remaining in the body's nose, pharyngeal cavity, trachea, and bronchus does not participate in lung gas exchange. This area is called the Physiological dead cavity. Its volume is about $150 \mathrm{ml}$. When people wear masks, the closed space formed between the mask and the face is called a structural geometry dead cavity. This space will retain a part of carbon dioxide produced by exhalation. Therefore, when people inhale the air again, they can only inhale the externally filtered air after inhaling this part of the carbon dioxide into the human body. The geometric dead cavity of the mask can reduce the concentration of oxygen in the air drawn into the lungs. Insufficient oxygen supply can damage various organs of the body and cause stress [9] [10]. The structural geometric dead cavity of the mask is shown in Figure 1.

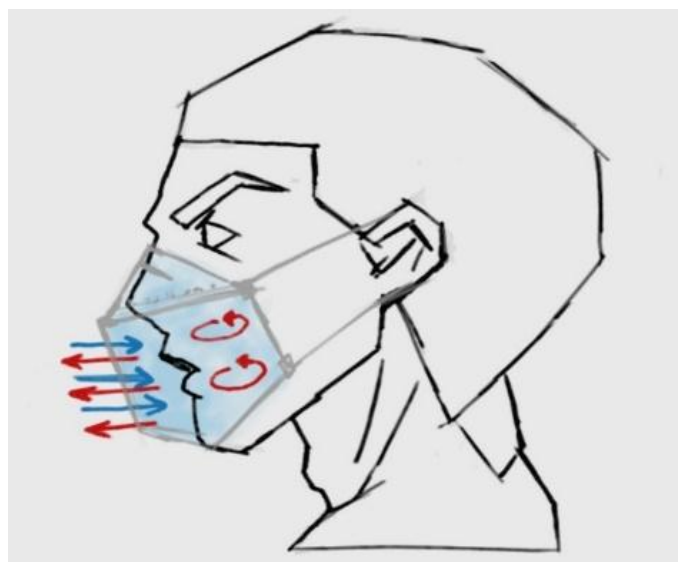

Figure 1. Structural geometric dead cavity of masks

\section{PRINCIPLE OF DEEP BREATHING METHOD TO RELIEVE MASK-INDUCED STRESS}

\subsection{Deep breathing method}

Control the breathing directly through the brain, slow down the breathing rhythm, inhale for 3 seconds, pause for 1 second, exhale for 4 seconds, and pause for 1 second. This completes the breathing and then repeats this activity. A person's normal breathing rate is 12 to 20 times per minute. Deep breathing needs to be reduced to about 8 times per minute, especially to increase the exhalation time [11].

\subsection{Respiratory rhythm is related to heart rate}

In neurology, the autonomic nerve of the human body includes the sympathetic nervous system and parasympathetic nervous system. The two mechanisms of action are completely opposite. Sympathetic nerves have a promoting effect, keeping the body alert, improving concentration, and reaching a state that can actively respond. In contrast, parasympathetic nerves have an inhibitory effect and are responsible for relaxing the body, preserving physical strength, promoting digestion, and initiating sleep [11] [12].

In fact, respiratory rhythm and heart rate are closely related, and deep breathing can strengthen the inhibitory and regulating effect of the autonomic nerve parasympathetic nerve, as to achieve soothing and relaxing effects. When a person inhales and expands the lungs, it will activate the stretch receptor on the lung organs to transmit signals to the brain and inhibit the sympathetic nerves. At this time, the role of the parasympathetic nerves will be strengthened and the human body will feel relaxed and soothed. In other words, the lungs expand during inhalation to suppress the sympathetic effect. Then the lung blood returns to the body during exhalation to strengthen the parasympathetic effect. The two mechanisms of inhibiting sympathetic nerves and strengthening parasympathetic nerves produce the same inhibitory effect. As a result, the body will relax. This process is just like that the speed of the car decreases depends on the driver closing the throttle and applying the brakes. Because each deep breath takes much longer than normal breaths, deep breaths can relieve stress better than normal breaths [13] [14] [15].

\subsection{Deep breathing reduces airway resistance $\left(\boldsymbol{R}_{\text {AW }}\right)$}

Deep breathing increases the pressure of the lungs $\left(P_{A}\right)$ by increasing the contractility of the lung muscles, so $\Delta P=$ $P_{A T M}-P_{A}$ decreases, that is, the pressure difference between the lungs and the outside becomes smaller. And deep breathing can speed up the air flow rate (v), so $v * S$ also increases, which reduces the airway resistance $\left(R_{A W}\right)$, so our breathing becomes easier, so psychological stress is reduced. In addition, the inhalation of oxygen and the exhaustion of exhaust gas are accelerated, and the oxygen concentration in the lungs is increased, which also solves the problem of hypoxia. 


\section{RELATIONSHIP BETWEEN HRV AND STRESS INDEX}

\subsection{Heart rate}

Heart rate is the speed of the heartbeat, which is measured by the number of contractions (beats) of the heart per minute (bpm) [16]. The normal resting adult human heart rate is 60 100 bpm. and heart rate is not constant, it can vary according to the body's physical needs. For example, sleep, exercise, stress, and anxiety can all affect heart rate. this is regulated by sympathetic and parasympathetic input to the sinoatrial node.

Heart rate is usually measured by ECG, Blood Pressure Measurement and Pulse Oximeter. From electrocardiogram can know that one pulse is generated for each heartbeat, and the interval between two pulse peaks represents the interval time between two heartbeats, it is called the NN interval. As shown in Figure 2, the NN interval is the distance between the peaks of two pulses. [17].

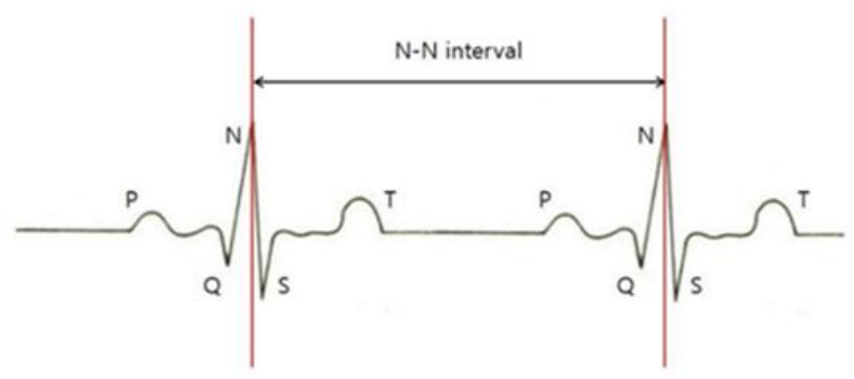

Figure 2. Electrocardiography (ECG Signal)

\subsection{Heart rate variability (HRV) and SDNN analysis methods}

Heart rate variability (HRV) is the physiological phenomenon of variation in the time interval between heartbeats. As shown in Figure 3, the length of each NN interval is different. HRV reflects the degree of variation of the NN interval. There is interest in HRV in the field of psychophysiology. For example, HRV is related to emotional arousal. HRV is the result of the common regulation and balance of sympathetic and parasympathetic nerves in the autonomic nervous system. Normal physiological activities will maintain a higher HRV change value. When the human body encounters stress, anxiety and other external environmental influences, it will inhibit the variation of heart rate, and reducing HRV. In other word, if a person physical and mental is healthy and normal, the heartbeat interval of this person is constantly variational, and the value is very large. When the person body is under stress, the inhibitory effect of the autonomic nerve will reduce this variation. So, the HRV will be very low [19] [18] [20].

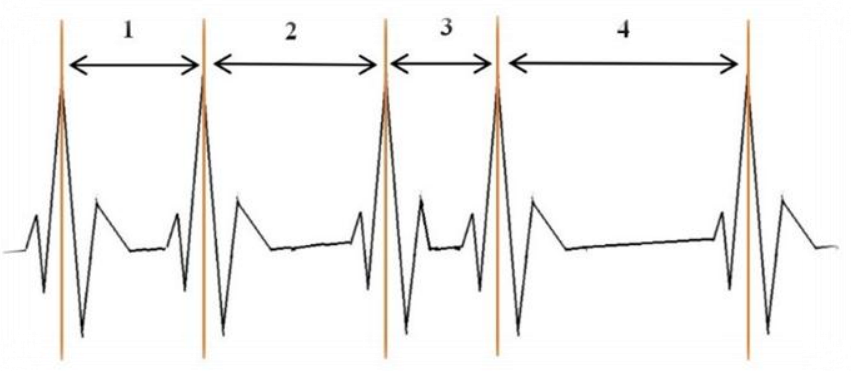

Figure 3. Interbeat Interval Series

Table 1. Correlation between SDNN and stress [20]

\begin{tabular}{|c|c|}
\hline Age & SDNN Reference \\
\hline $10 \sim 40$ & $\begin{array}{l}>\text { 50: Great, Autonomic nerve control and stress coping ability } \\
\text { 35 50: Normal, Autonomic nerve control and coping abilities are normal } \\
\text { 20 35: Low, Stress Disease Risk \& autonomic nerve weakness } \\
\text { < 20: Very low, Autonomic nerve high risk of chronic stress related illness }\end{array}$ \\
\hline $50 \sim 60$ & $\begin{array}{l}>40 \text { : Great } \\
\text { 15 20: Low }\end{array}$ \\
\hline
\end{tabular}

There are many methods for HRV analysis. SDNN is a commonly used time domain method, the standard deviation of NN intervals. This method calculates the standard deviation of the NN interval. Because the standard deviation can reflect the degree of discreteness of the data. It can be expressed as the greater the standard deviation, the greater the deviation of these 
values from the average. Therefore, SDNN can indicate the degree of time interval change between heartbeats [22] [23]. The following is the calculation formula of SDNN.

$$
\begin{aligned}
& \mathrm{N}-\mathrm{N} \text { Interval }(\mathrm{ms})=\frac{60000}{\text { Heart Rate }(\text { bpm })}, \\
& \operatorname{SDNN}(\sigma)=\sqrt{\frac{\sum_{k=1}^{N}\left(x_{k}-\mu\right)^{2}}{N}},
\end{aligned}
$$

\section{EXPERIMENTS AND RESULTS}

The subjects were 20 healthy men and women of different ages. The measurement environment is in a quiet office. Equipment includes: KF94 mask and Pulse Oximeter. Measuring instruments and measurement data format are shown in Figure 4. First measure the heart rate for three minutes of normal breathing while wearing a mask, then measure the heart rate for three minutes of deep breathing while wearing a mask. During the measurement, all subjects remain still and calm inside, and then performed the SDNN calculation on MATLAB to obtain the SDNN value, and finally compared the SDNN values measured in the two cases. The experimental process is shown in Figure 5.

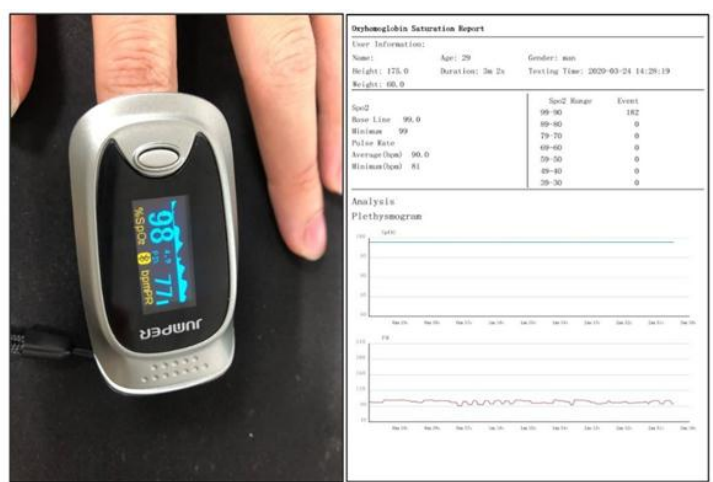

Figure 4. Pulse Oximeter and measurement data format

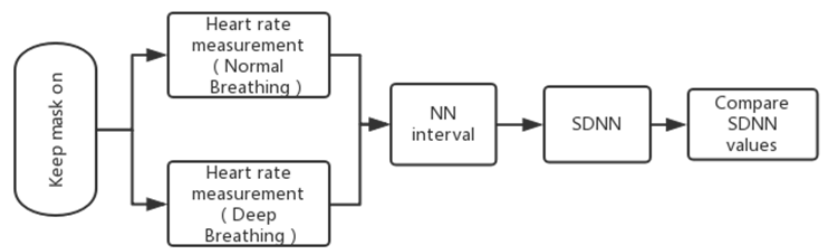

Figure 5. SDNN measurement and comparison

Figure 6 is a graph of the change in NN intervals of person 4 normal breathing (left) and deep breathing(right) under a mask. It is easy to find that the deviation between mean and NN interval is large during take deep breathing with a mask. It means that the degree of change in the $\mathrm{NN}$ interval during deep breathing is greater than that of normal breathing, that is, the SDNN of deep breathing value is greater than normal breathing. So, the stress is lower when person 4 take a deep breath.

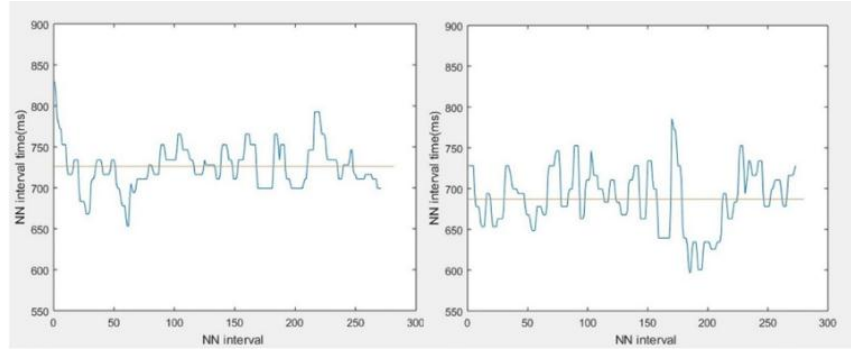

Figure 6. The change in NN intervals of person 4 normal breathing (left) and deep breathing(right) under a mask

Table 2 shows that the average heart rate of 20 subjects who participated in the heart rate test, SDNN experimental results, the difference between normal breathing and deep breathing $\mathrm{SDNN}(\triangle \mathrm{SDNN}=\mathrm{S} 2-\mathrm{S} 1)$. Figure 7 is a graph of $\triangle \mathrm{SDNN}$ values for 20 subjects.

Table 2. Results of Heart Rate measurement

\begin{tabular}{|c|c|c|c|c|c|}
\hline \multirow{2}{*}{ Subjects } & \multicolumn{2}{|c|}{ Normal Breathing (Mask on ) } & \multicolumn{2}{c|}{ Deep Breathing ( Mask on ) } & \multirow{2}{*}{$\begin{array}{c}\text { ASDNN } \\
\text { : S2-S1 }\end{array}$} \\
\cline { 2 - 5 } & $\begin{array}{c}\text { Average heart } \\
\text { rate(bpm) }\end{array}$ & SDNN 1(S1) & $\begin{array}{c}\text { Average heart } \\
\text { rate(bpm) }\end{array}$ & SDNN 2(S2) & \\
\hline $\mathbf{1}$ & 90 & 22.04 & 90 & 26.55 & $\mathbf{4 . 5 1}$ \\
\hline $\mathbf{2}$ & 84 & 35.43 & 90 & 34.58 & $\mathbf{- 0 . 8 5}$ \\
\hline $\mathbf{3}$ & 85 & 20.86 & 90 & 34.33 & $\mathbf{1 3 . 4 7}$ \\
\hline $\mathbf{4}$ & 84 & 26.53 & 88 & 36.80 & $\mathbf{1 0 . 2 7}$ \\
\hline $\mathbf{5}$ & 83 & 36.97 & 89 & 35.34 & $\mathbf{- 1 . 6 3}$ \\
\hline $\mathbf{6}$ & 78 & 26.88 & 89 & 45.04 & $\mathbf{1 8 . 1 6}$ \\
\hline $\mathbf{7}$ & 78 & 33.05 & 79 & 52.81 & $\mathbf{1 9 . 7 6}$ \\
\hline
\end{tabular}




\begin{tabular}{|c|c|c|c|c|c|}
\hline \multirow{2}{*}{ Subjects } & \multicolumn{2}{|c|}{ Normal Breathing ( Mask on ) } & \multicolumn{2}{|c|}{ Deep Breathing ( Mask on ) } & \multirow{2}{*}{$\begin{array}{l}\Delta S D N N \\
=\text { S2-S1 }\end{array}$} \\
\hline & $\begin{array}{l}\text { Average heart } \\
\text { rate(bpm) }\end{array}$ & SDNN 1(S1) & $\begin{array}{l}\text { Average heart } \\
\text { rate(bpm) }\end{array}$ & SDNN 2(S2) & \\
\hline 8 & 72 & 46.19 & 84 & 48.42 & 2.23 \\
\hline 9 & 85 & 25.99 & 93 & 38.39 & 12.4 \\
\hline 10 & 81 & 31.23 & 87 & 42.21 & 10.98 \\
\hline 11 & 77 & 28.75 & 88 & 31.64 & 2.89 \\
\hline 12 & 79 & 27.21 & 89 & 40.33 & 13.12 \\
\hline 13 & 80 & 22.34 & 77 & 59.78 & 37.44 \\
\hline 14 & 77 & 28.89 & 76 & 43.85 & 14.96 \\
\hline 15 & 75 & 31.24 & 77 & 55.52 & 24.28 \\
\hline 16 & 96 & 17.20 & 95 & 29.29 & 12.09 \\
\hline 17 & 85 & 37.72 & 86 & 40.01 & 2.29 \\
\hline 18 & 73 & 23.19 & 78 & 35.61 & 12.42 \\
\hline 19 & 66 & 25.68 & 65 & 29.53 & 3.85 \\
\hline 20 & 81 & 30.05 & 87 & 39.43 & 9.38 \\
\hline
\end{tabular}

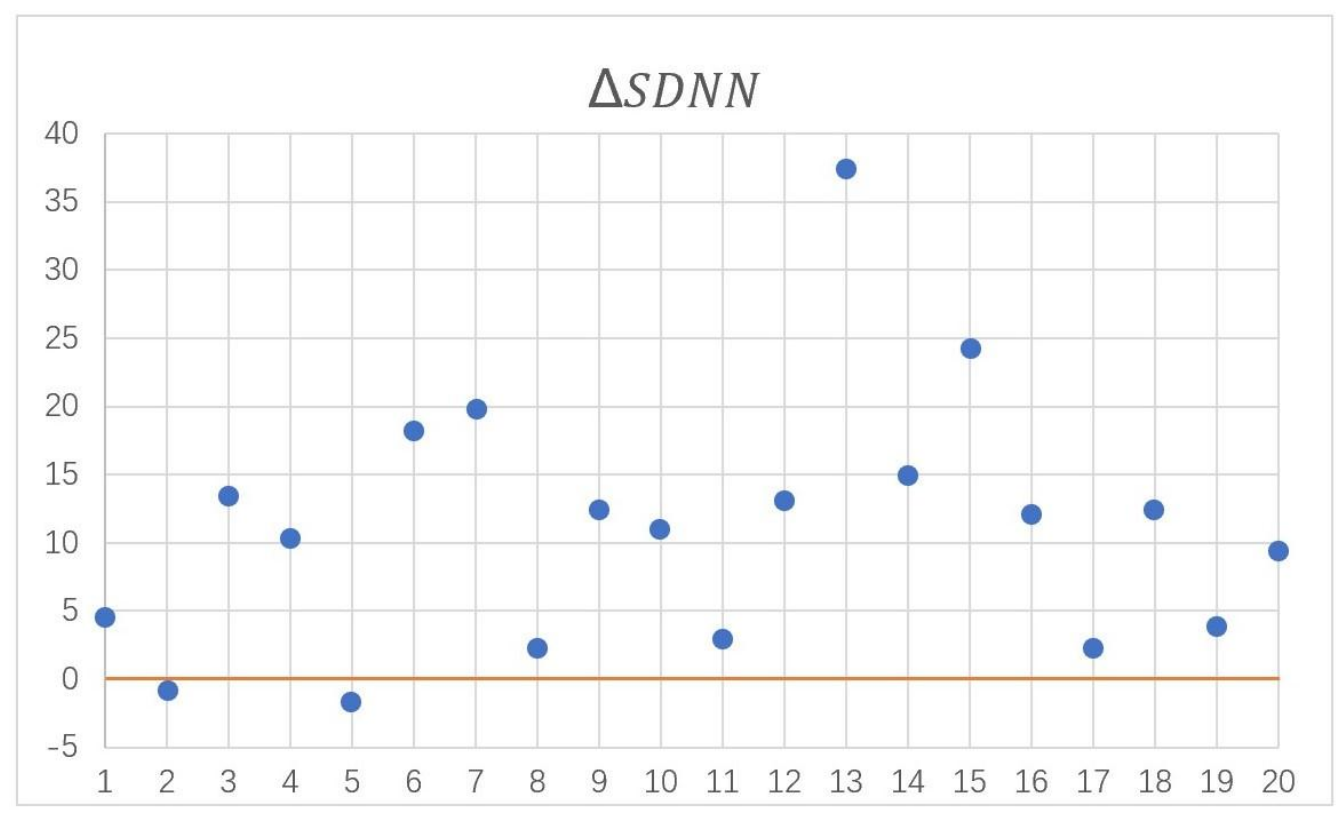

Figure 7. $\triangle$ SDNN values of 20 subjects

According to compare all the experimental results in Table 2, it is found that after using the deep breathing method, 18 of 20 people had significantly increased SDNN values. In addition, only two of them had a small decrease in SDNN value. The number of SDNN increase accounts for $90 \%$ of the total number of people, and among all the people who increase the SDNN, the average increase of SDNN is 12.47 , this also means that the stress is greatly reduced. As Figure 7, this result strongly represent that the deep breathing method can relieve the stress induced by wearing a mask very well.

\section{CONCLUSION}

The problem of hypoxia and stress caused by wearing protective masks for a long time has proven. With the global epidemic of COVID 19, the number of people choosing to protect themselves by wearing masks has increased dramatically. In this case, it is found that deep breathing is a great method in order to reduce the pressure of wearing a mask.

In this paper, the correlation between HRV and stress was used to perform heart rate measurement experiments and SDNN analysis. Comparing the results of the two cases of experiments, it was found that the Deep Breathing Method 
reduced the stress induced by wearing a mask for most subjects, and the stress have a great drop. Therefore, the Deep Breathing Method is very effective and simple for reducing the negative side caused by wearing a mask. Of course, this method is not only effective when wearing a mask. When people take off their masks to rest, using deep breathing can also help people reduce stress more quickly.

Therefore, this paper suggests that when wearing a mask for more than half an hour, wash and disinfect hands first, then take off the mask and take a deep breath for three minutes to quickly release the stress. If the condition does not allow people to take off the mask, wear a mask and take a deep breath for three to five minutes to relieve stress and hypoxia.

This method makes it possible for people who have to wear masks for a long time can relieve the stress without removing them, which is good for their health. Because of its simplicity and ease of operation, it is easier to be accepted by the public. This simple and effective method mentioned in this study may be promoted and can help more people protect their health.

\section{REFERENCE}

[1] Oxygen deficiency-confined [https://mydaum00.tistory.com/m/300].

space.

[2] Olivier Coste, Pascal Van Beers, André Bogdan \&Prof. Yvan Touitou. "Human Immune Circadian System in Prolonged Mild Hypoxia during Simulated Flights". Pages 87-98 | Received 11 Sep 2006, Accepted 18 Sep 2006, Published online: 07 Jul 2009.

[3] Segerstrom SC, Miller GE (2004) "Psychological stress and the human immune system: a meta-analytic study of 30 years of inquiry". Psychol Bull 130:601-630

[4] Zorrilla EP, Luborsky L, McKay JR et al (2001) "The relationship of depression and stressors to immunological assays: a meta-analytic review ". Brain Behav Immun 15:199-226.

[5] Bong-Young Kim, Ik-Soo Ahn and Myung-Jin Bae. " A Study on Lung Function Activation of Sound Necklace".Journal of Engineering and Applied Sciences (2018)Volume: 13 , Issue: 3, Page

[6] [6] Zhixing Tian, Bong-Young Kim, Myung-Jin Bae. "A Study on the Effect of Wearing Masks on Stress Response". International Journal of Engineering Research and Technology 13(3), To be published.

[7] Zhixing Tian, Bong-Young Kim, Myung-Jin Bae. "A study on the Improvement of Klaxon Sound". International Journal of Engineering Research and Technology Volume 12, Issue 12, 2019, Pages 24402445.

[8] Kirkby, J.; et al. (2010). "Reference equations for specific airway resistance in children: The Asthma UK initiative". European Respiratory Journal. 36 (3): 622-629.

[9] Test method for airflow resistance of respiration of filtering particle respirator" Published by China National Bureau of Standards , 1986-04-09.
[10] Central Daily News. " If you lack oxygen, you'll be happy hormones. $\downarrow$ If you take an oxygen shower, you will feel depressed and anxious [https://news.joins.com/article/23008248].

[11] Ravinder Jerath *, John W. Edry, Vernon A. Barnes, Vandna Jerath. "Physiology of long pranayamic breathing: Neural respiratory elements may provide a mechanism that explains how slow deep breathing shifts the autonomic nervous system ". Medical Hypotheses Volume 67, Issue 3, 2006, Pages 566-571.

[12] Bong-Young Kim, Zhixing Tian, Myung-Jin Bae. "A study on the causes of revenge psychology of Klaxon sound". International Journal of Engineering Research and Technology Volume 12, Issue 12, 2019, Pages 2947 2952.

[13] ROBERT W. SHIELDS, JR, MD. "Heart rate variability with deep breathing as a clinical test of cardiovagal function ". Heart rate variability with deep breathing as a clinical test of cardiovagal function.

[14] Pal G, Velkumary S, Madanmohan. "Effect of short-term practice of breathing exercises on autonomic functions in normal human volunteers ". Indian $\mathrm{J}$ Med Res 2004;120(2):115-21.

[15] 360 Personal Library. "Uncovering Secrets-Deep Breathing Can Relieve Stress" [http://www.360doc.com/content/17/0516/13/18915470_ 654385125.shtml].

[16] "All About Heart Rate (Pulse)". American Heart Association. 22 Aug 2017. Retrieved 25 Jan 2018.

[17] Jönsson P (January 2007). "Respiratory sinus arrhythmia as a function of state anxiety in healthy individuals", International Journal of Psychophysiology. 63 (1): 48-54

[18] CAMM, A. John, et al. "Heart rate variability: standards of measurement, physiological interpretation and clinical use". Task Force of the European Society of Cardiology and the North American Society of Pacing and Electrophysiology Circulation. Vol.93, No.5, pp.10431065, 1996.

[19] Appelhans, Bradley M.; Luecken, Linda J. (September 2006). "Heart Rate Variability as an Index of Regulated Emotional Responding". Review of General Psychology. 10 (3): 229-240.

[20] Bong-Young Kim, Myung-Jin Bae."A study on the effect of jogging on stress reduction". International Journal of Engineering Research and Technology 11(9). pp. 13631370.

[21] Bong-Young Kim, Myung-Jin Bae. "A study on the stress reduction effect of reading aloud the book using HRV". International Journal of Engineering Research and Technology Volume 12, Issue 9, 2019, Pages 1457-1461.

[22] Winbo, A., Paterson, D.J. " The Brain-Heart Connection in Sympathetically Triggered Inherited Arrhythmia Syndromes ". Heart Lung and Circulation Volume 29, Issue 4, April 2020, Pages 529-537.

[23] Koolhaas J, et al. (2011). "Stress revisited: A critical evaluation of the stress concept". Neuroscience and Biobehavioral Reviews. 35 (5): 1291-1301 